\title{
RECURRENCE DETERMINISM AND LI-YORKE CHAOS FOR INTERVAL MAPS
}

\author{
VLADIMÍR ŠPITALSKÝ
}

\begin{abstract}
Recurrence determinism, one of the fundamental characteristics of recurrence quantification analysis, measures predictability of a trajectory of a dynamical system. It is tightly connected with the conditional probability that, given a recurrence, following states of the trajectory will be recurrences.

In this paper we study recurrence determinism of interval dynamical systems. We show that recurrence determinism distinguishes three main types of $\omega$-limit sets of zero entropy maps: finite, solenoidal without non-separable points, and solenoidal with non-separable points. As a corollary we obtain characterizations of strongly non-chaotic and Li-Yorke (non-)chaotic interval maps via recurrence determinism. For strongly non-chaotic maps, recurrence determinism is always equal to one. Li-Yorke non-chaotic interval maps are those for which recurrence determinism is always positive. Finally, Li-Yorke chaos implies the existence of a Cantor set of points with zero determinism.
\end{abstract}

\section{INTRODUCTION}

Recurrence plots, introduced by Eckmann et al. [3], provide a visual representation of trajectories of dynamical systems, which is well-suited for data analysis. Quantitative study of recurrence plots, called recurrence quantification analysis [17, has been successfully applied in many areas of science; see [16 for a comprehensive overview of the subject. One of its basic and most used characteristics is called determinism. A slightly modified notion, which will be called here recurrence determinism and $\operatorname{denoted~} \operatorname{rdet}_{m}(x, \varepsilon)$, is tightly connected with the conditional probability that the next $m$ states of the trajectory of a point $x$ will be $\varepsilon$-recurrences given that the current state is an $\varepsilon$-recurrence; see Section 3.2 for details. Thus if recurrence determinism is high, upon encountering a recurrence we can successfully predict subsequent $m$ states of the trajectory.

Asymptotic properties of various quantitative recurrence characteristics were studied in 4, 15, 18, 5, 7, 6, 10, among others. The purpose of this paper is to show that behavior of recurrence determinism $\operatorname{rdet}_{\infty}(x, \varepsilon)$ for small $\varepsilon$ is able to distinguish among various types of interval dynamics. In [14, Theorem B] it was proved that topological entropy is the supremum of local correlation entropies. As a consequence we have

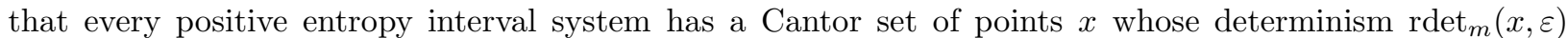
converges to zero exponentially fast as $m \rightarrow \infty$, and thus $\operatorname{rdet}_{\infty}(x, \varepsilon)=0$ for all sufficiently small $\varepsilon>0$. For

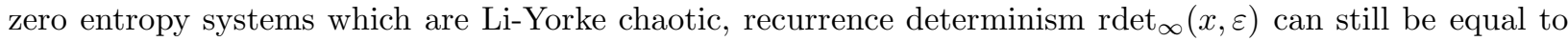
zero. On the other hand, interval maps which are not Li-Yorke chaotic have recurrence determinism always positive. Finally, if recurrence determinism is equal to one for every point of the interval, then the system is strongly non-chaotic, that is, all $\omega$-limit sets of it are finite. All these results are summarized in the following theorem.

Theorem 1. Let $f: I \rightarrow I$ be continuous. Then

(1) $f$ is strongly non-chaotic if and only if $\operatorname{rdet}_{\infty}(x, \varepsilon)=1$ for every $x \in I$ and every sufficiently small $\varepsilon>0$

(2) $f$ is Li-Yorke non-chaotic if and only if $\underline{\operatorname{rdet}}_{\infty}(x, \varepsilon)>0$ for every $x \in I$ and every sufficiently small $\varepsilon>0$

(3) $f$ is Li-Yorke chaotic if and only if there is a Cantor set $C$ such that $\operatorname{rdet}_{\infty}(x, \varepsilon)=0$ for every $x \in C$ and every sufficiently small $\varepsilon>0$.

The next theorem states that, for any fixed point $x$, behavior of $\operatorname{rdet}_{\infty}(x, \varepsilon)$ for small $\varepsilon>0$ depends on the type of the $\omega$-limit set $\omega_{f}(x)$ of $x$. For the corresponding definitions see Section 2

2010 Mathematics Subject Classification. Primary 37E05; Secondary 37B05, 54H20.

Key words and phrases. recurrence determinism, interval map, Li-Yorke chaos. 
Theorem 2. Let $f: I \rightarrow I$ be a continuous map with zero entropy and $x \in I$. Then

(1) $\omega_{f}(x)$ is finite if and only if

$$
\operatorname{rdet}_{\infty}(x, \varepsilon)=1 \quad \text { for every sufficiently small } \varepsilon>0
$$

(2) $\omega_{f}(x)$ is solenoidal and does not contain non-separable points if and only if

$$
\underline{\operatorname{rdet}}_{\infty}(x, \varepsilon)>0 \quad \text { for every } \varepsilon>0
$$

and

$$
\liminf _{\varepsilon \rightarrow 0} \overline{\operatorname{rdet}}_{\infty}(x, \varepsilon)<1
$$

(3) $\omega_{f}(x)$ is solenoidal and contains non-separable points if and only if

$$
\operatorname{rdet}_{\infty}(x, \varepsilon)=0 \quad \text { for every sufficiently small } \varepsilon>0 .
$$

Note that the conditions from Theorem 2(2) cannot be strengthened. As was shown in [8, Theorem 4.12], determinism of a Li-Yorke non-chaotic map can be strictly smaller than 1 for every small $\varepsilon$, even with $\limsup _{\varepsilon \rightarrow 0} \overline{\operatorname{rdet}}_{\infty}(x, \varepsilon)<1$. On the other hand, our final result asserts that, for a Li-Yorke non-chaotic map with an infinite $\omega$-limit set, there are no uniform boundaries for recurrence determinism, that is, we cannot find $\alpha$ and $\beta$ such that $0<\alpha \leq \underline{\operatorname{rdet}}_{\infty}(x, \varepsilon) \leq \overline{\operatorname{rdet}}_{\infty}(x, \varepsilon) \leq \beta<1$ for every sufficiently small $\varepsilon>0$.

Theorem 3. There is a Li-Yorke non-chaotic map $f: I \rightarrow I$ of type $2^{\infty}$ with unique infinite minimal set $C$, and sequences $\left(\varepsilon_{k}\right)_{k},\left(\varepsilon_{k}^{\prime}\right)_{k}$ decreasing to zero such that, for every $x \in C, \operatorname{rdet}_{\infty}\left(x, \varepsilon_{k}\right)=1$ for every $k$ and $\lim _{k} \overline{\operatorname{rdet}}_{\infty}\left(x, \varepsilon_{k}^{\prime}\right)=0$. Consequently,

$$
\limsup _{\varepsilon \rightarrow 0} \underline{\operatorname{rdet}}_{\infty}(x, \varepsilon)=1 \quad \text { and } \quad \liminf _{\varepsilon \rightarrow 0} \overline{\operatorname{rdet}}_{\infty}(x, \varepsilon)=0 \quad \text { for every } x \in C .
$$

In the following two sections we recall necessary notions and facts and we prove some preliminary lemmas. Theorems 13 are then proved in Sections 4 and 5 .

\section{Preliminaries}

The sets of all, positive, non-negative integers are denoted by $\mathbb{Z}, \mathbb{N}, \mathbb{N}_{0}$, respectively. Let $I$ denote the unit interval $[0,1]$ equipped with the Euclidean metric $\varrho$, and $\lambda$ denote the Lebesgue measure on $I$. If $J, J^{\prime}$ are intervals, by $J<J^{\prime}\left(J \leq J^{\prime}\right)$ we mean that $y<y^{\prime}\left(y \leq y^{\prime}\right)$ for every $y \in J$ and $y^{\prime} \in J^{\prime}$; analogously we define $a<J$ and $a \leq J$ for any real number $a$. If $A$ is a subset of a topological space, its boundary is denoted by $\partial A$. The cardinality of a finite set $A$ is denoted by \# $A$.

If no confusion can arise, a set $\{m, m+1, \ldots, n\}$ of consecutive integers is denoted by $[m, n]$ or by $[m, n+1)$. If $\alpha=\alpha_{0} \alpha_{1} \ldots$ is a (finite or infinite) sequence and $0 \leq k<l$ are integers, by $\alpha_{[k, l)}$ or $\alpha[k, l)$ we mean $\alpha_{k} \alpha_{k+1} \ldots \alpha_{l-1}$.

2.1. Dynamical systems. A dynamical system is a pair $(X, f)$, where $X$ is a compact metric space with a metric $\varrho$, and $f: X \rightarrow X$ is a continuous map. A nonempty subset $Y$ of $X$ is called p-periodic (for some $p \in \mathbb{N})$ if $f^{i}(Y), 0 \leq i<p$, are pairwise disjoint and $f^{p}(Y)=Y$. Note that if $Y$ is $p$-periodic and $Y^{\prime} \subseteq Y$ is $p^{\prime}$-periodic then $p^{\prime}$ is a multiple of $p$.

A point $x \in X$ is called $p$-periodic or just periodic if $\{x\}$ is $p$-periodic. It is called non-wandering if for every neighborhood $U$ of $x$ there is $n>0$ such that $f^{n}(U) \cap U \neq \emptyset$. The sets of all periodic and all non-wandering points of $(X, f)$ are denoted by $\operatorname{Per}(f)$ and $\Omega(f)$, respectively. The orbit of $x$ is the set $\operatorname{Orb}_{f}(x)=\left\{f^{n}(x): n \in \mathbb{N}_{0}\right\}$. The $\omega$-limit set of $x$, that is, the set of all limit points of the trajectory $\left(f^{n}(x)\right)_{n \in \mathbb{N}_{0}}$, is denoted by $\omega_{f}(x)$.

Let $f: X \rightarrow X$ be a dynamical system. A pair $(y, z)$ of points from $X$ is called a scrambled pair if

$$
\liminf _{n \rightarrow \infty} \varrho\left(f^{n}(y), f^{n}(z)\right)=0 \quad \text { and } \quad \limsup _{n \rightarrow \infty} \varrho\left(f^{n}(y), f^{n}(z)\right)>0 .
$$

A set $S \subseteq X$ is called scrambled if $(y, z)$ is a scrambled pair for every $y \neq z$ from $S$. The dynamical system $(X, f)$ is Li-Yorke chaotic if there exists an uncountable scrambled set $S \subseteq X$.

Let $f: I \rightarrow I$ be continuous. Following [13, Definition 2.1], we say that points $y, z \in I$ are separable if there are disjoint periodic intervals $J_{y}, J_{z}$ such that $y \in J_{y}$ and $z \in J_{z}$. If $y, z$ are distinct and not separable, we say that they are non-separable. By [13, Theorem 2.2] (see also [11, Theorem 5.21]), a zero 
entropy interval map is Li-Yorke chaotic if and only if there exists an infinite $\omega$-limit set containing two non-separable points.

2.2. Solenoidal $\omega$-limit sets. Let $f: I \rightarrow I$ be a continuous map and $x \in I$ be such that $\omega_{f}(x)$ is solenoidal. That is (see [1, p. 4]), there are a sequence of integers $2 \leq p_{0}<p_{1}<\ldots$ and a sequence of non-degenerate closed intervals $J_{0} \supseteq J_{1} \supseteq \ldots$ such that every $J_{k}$ is $p_{k}$-periodic and $\omega_{f}(x) \subseteq Q$, where

$$
Q=\bigcap_{t=0}^{\infty} Q_{t}, \quad Q_{t}=\bigsqcup_{i=0}^{p_{t}-1} f^{i}\left(J_{t}\right) .
$$

Put $q_{0}=p_{0}$ and $q_{t}=p_{t} / p_{t-1}$ for $t \geq 1$. Define

$$
\Sigma=\prod_{i=0}^{\infty}\left\{0, \ldots, q_{i}-1\right\}, \quad \mathcal{A}^{t}=\prod_{i=0}^{t-1}\left\{0, \ldots, q_{i}-1\right\} \quad(t \geq 1) ;
$$

every element $a=a_{0} a_{1} \ldots a_{t-1}$ of $\mathcal{A}^{t}$ is called a word and the length of it is $|a|=t$. Define also $\mathcal{A}^{0}=\{o\}$ (a singleton set containing the empty word $o$ ) and $\mathcal{A}^{*}=\bigsqcup_{t \geq 0} \mathcal{A}^{t}$. Let $\pi_{t}: \Sigma \rightarrow \mathcal{A}^{t}(t \geq 0)$ be the natural projection onto the first $t$ coordinates. For $a \in \mathcal{A}^{t}$ denote by $[a]$ the set of all sequences $\alpha \in \Sigma$ and all words $b \in \mathcal{A}^{*}$ starting with $a$ (i.e. $\pi_{t}(\alpha)=\pi_{t}(b)=a$ ).

On $\Sigma$ and on every $\mathcal{A}^{t}$ define addition in a natural way with carry from left to right; the sets $\Sigma$ and $\mathcal{A}^{t}$ equipped with this operation are abelian groups. Identify $10^{\infty} \in \Sigma$ and every $10^{t-1} \in \mathcal{A}^{t}(t \geq 1)$ with integer 1 , and inductively define $\alpha+n, a+n$ for $\alpha \in \Sigma, a \in \mathcal{A}^{t}$, and $n \in \mathbb{Z}$.

For $t \geq 0$ write

$$
Q_{t}=\bigsqcup_{a \in \mathcal{A}^{t}} K_{a}, \quad \text { where } K_{0^{t}+i}=f^{i}\left(J_{t}\right) \text { for every } i \in\left[0, p_{t}\right) .
$$

Notice that every $K_{a}\left(a \in \mathcal{A}^{t}\right)$ is a non-degenerate closed $p_{t}$-periodic interval $\left[y_{a}, z_{a}\right]$, and $K_{b} \subseteq K_{a}$ for every $b \in[a]$. We can also write

$$
Q=\bigsqcup_{\alpha \in \Sigma} K_{\alpha}, \quad \text { where } K_{\alpha}=\bigcap_{t=0}^{\infty} K_{\pi_{t}(\alpha)} \text { for every } \alpha \in \Sigma .
$$

Here, every $K_{\alpha}$ is either a singleton $\left\{y_{\alpha}\right\}$ or a non-degenerate closed interval $\left[y_{\alpha}, z_{\alpha}\right]$.

The following is a direct consequence of [1, Theorem 3.1].

Lemma 4. Let $f: I \rightarrow I$ be continuous and $x \in I$. Let $\omega_{f}(x)$ be solenoidal and $Q$ be as defined above. Let $C \subseteq Q \cap \overline{\operatorname{Per}}(f)$ be the (Cantor) set of all limit points of $Q \cap \Omega(f)$. Then the following assertions are true:

(1) if $y \in Q$ then $\omega_{f}(y)=C$;

(2) if $y \in I$ and $\omega_{f}(y) \cap Q \neq \emptyset$ then $C \subseteq \omega_{f}(y) \subseteq Q \cap \Omega(f)$; moreover, for every $\alpha \in \Sigma$,

$$
\emptyset \neq C \cap K_{\alpha} \subseteq \omega_{f}(y) \cap K_{\alpha} \subseteq \Omega(f) \cap K_{\alpha} \subseteq \partial K_{\alpha} .
$$

Since every $Q_{t}$ is $f$-invariant and has finite boundary, we immediately have the following lemma.

Lemma 5. Let $\omega_{f}(x)$ be solenoidal and $Q=\bigcap Q_{t}$ be as defined above. Then for every $t$ there is $n_{0}$ such that $f^{n}(x) \in Q_{t}$ for every $n \geq n_{0}$.

The next lemma follows from [1, Theorems 5.4 and 4.1(d)] and [13; see also [11, Proposition 5.24].

Lemma 6. Let $f: I \rightarrow I$ have zero entropy and $x \in I$. Then one of the two cases happens:

(1) $\omega_{f}(x)$ is finite (and hence a periodic orbit);

(2) $\omega_{f}(x)$ is solenoidal.

If (2) is true then $Q$ is 2-adic, that is, one can choose $p_{t}=2^{t}$ for every $t$.

Lemma 7. Let $f: I \rightarrow I$ have zero entropy and $x \in I$ be such that $\omega_{f}(x)$ is solenoidal. Then, for every $y \neq z$ from $\omega_{f}(x), y$ and $z$ are non-separable if and only if there is $\alpha \in \Sigma$ such that $K_{\alpha}$ is non-degenerate and $y, z$ are the endpoints of $K_{\alpha}$.

Proof. The lemma directly follows from [11, Lemma 5.26], which asserts that distinct $y, z \in \omega_{f}(x)$ are non-separable if and only if for every $t \geq 1$ there is $a^{(t)} \in \mathcal{A}^{t}$ such that $y, z \in K_{a^{(t)}}$. 


\section{Correlation SUM AND RECURRENCE DETERMINISM}

3.1. Correlation sum. Let $(X, \varrho)$ be a compact metric space and $f: X \rightarrow X$ be a continuous map. For $x \in X, \varepsilon>0$, and $n \in \mathbb{N}$, the correlation sum is defined by

$$
C_{\varrho}(x, n, \varepsilon)=\frac{1}{n^{2}} \cdot \#\left\{(i, j): 0 \leq i, j<n, \varrho\left(f^{i}(x), f^{j}(x)\right) \leq \varepsilon\right\} .
$$

The lower and upper asymptotic correlation sums are

$$
\underline{c}_{\varrho}(x, \varepsilon)=\liminf _{n \rightarrow \infty} C_{\varrho}(x, n, \varepsilon), \quad \bar{c}_{\varrho}(x, \varepsilon)=\limsup _{n \rightarrow \infty} C_{\varrho}(x, n, \varepsilon) .
$$

Note that (asymptotic) correlation sums are numbers from the unit interval $[0,1]$, and are equal to 1 for $\varepsilon \geq \operatorname{diam} X$. Note also that (asymptotic) correlation sums are non-decreasing functions of $\varepsilon$.

Let $m \geq 1$ be an integer. Bowen's metric $\varrho_{m}=\varrho_{m}^{f}$ is given by

$$
\varrho_{m}(x, y)=\max \left\{\varrho\left(f^{i}(x), f^{i}(y)\right): 0 \leq i<m\right\} \quad \text { for every } x, y \in X .
$$

It is a metric on $X$ compatible with the topology of $X$, thus we may define (asymptotic) correlation sum with respect to this metric. For abbreviation, we write $C_{m}(x, n, \varepsilon), \underline{c}_{m}(x, \varepsilon)$, and $\bar{c}_{m}(x, \varepsilon)$ instead of $C_{\varrho_{m}}(x, n, \varepsilon)$, $\underline{c}_{\varrho_{m}}(x, \varepsilon)$, and $\bar{c}_{\varrho_{m}}(x, \varepsilon)$, respectively.

Since $\varrho_{m} \leq \varrho_{m+1}$ for every $m$, we have that $C_{m}(x, n, \varepsilon), \underline{c}_{m}(x, \varepsilon)$, and $\bar{c}_{m}(x, \varepsilon)$ are non-increasing functions of $m$. Denote the corresponding limits, as $m$ approaches infinity, by $C_{\infty}(x, n, \varepsilon), \underline{c}_{\infty}(x, \varepsilon)$, and $\bar{c}_{\infty}(x, \varepsilon)$, respectively. Note that $C_{\infty}(x, n, \varepsilon)=C_{\varrho_{\infty}}(x, n, \varepsilon)$, where $\varrho_{\infty}$ is a metric given by $\varrho_{\infty}(x, y)=$ $\sup \left\{\varrho\left(f^{i}(x), f^{i}(y)\right): 0 \leq i<\infty\right\}$. In general, however, the metric $\varrho_{\infty}$ need not be compatible with the topology of $X$; e.g. for an expansive system $(X, f)$ the metric $\varrho$ is always discrete.

The following is Lemma 8 from [14].

Lemma 8. Let $X$ be a compact metric space and $\varepsilon>0$. Then there is $\eta \in(0,1)$ such that, for every continuous map $f: X \rightarrow X$ and every $x \in X$,

$$
\bar{c}_{m}(x, \varepsilon) \geq \underline{c}_{m}(x, \varepsilon) \geq \eta^{m} \quad \text { for every } m \in \mathbb{N} .
$$

3.2. Recurrence determinism. For $x \in X, \varepsilon>0, m \in \mathbb{N} \cup\{\infty\}$, and $n \geq 1$, define the recurrence m-determinism by

$$
\operatorname{rdet}_{m}(x, n, \varepsilon)=\frac{C_{m}(x, n, \varepsilon)}{C_{1}(x, n, \varepsilon)}
$$

and the upper and lower asymptotic recurrence $m$-determinism by

$$
\underline{\operatorname{rdet}}_{m}(x, \varepsilon)=\liminf _{n \rightarrow \infty} \operatorname{rdet}_{m}(x, n, \varepsilon), \quad \overline{\operatorname{rdet}}_{m}(x, \varepsilon)=\limsup _{n \rightarrow \infty} \operatorname{rdet}_{m}(x, n, \varepsilon) .
$$

If $\underline{\operatorname{rdet}}_{m}(x, \varepsilon)=\overline{\operatorname{rdet}}_{m}(x, \varepsilon)$ we say that the recurrence $m$-determinism exist and we denote the common value by $\operatorname{rdet}_{m}(x, \varepsilon)$; analogously for $c_{m}(x, \varepsilon)$.

Remark 9 (RQA-determinism). Note that, for finite $m$, the definition of recurrence determinism slightly differs from that used in recurrence quantification analysis. Since here we deal with embedding dimension 1, RQA-determinism is $\operatorname{DET}_{m}(x, n, \varepsilon)=m \cdot \operatorname{rdet}_{m}(x, n, \varepsilon)-(m-1) \cdot \operatorname{rdet}_{m+1}(x, n, \varepsilon)$ by [7, Proposition 1], see also [12, Theorem 2]. However, for $m=\infty$ it holds that $\operatorname{DET}_{\infty}(x, n, \varepsilon)=\operatorname{rdet}_{\infty}(x, n, \varepsilon)$ by [8, Lemma 2.1].

Remark 10 (Recurrence determinism as a conditional probability). Asymptotic recurrence determinism is (for typical $x$ and $\varepsilon$ ) equal to the conditional probability that the following $m$ states of the trajectory will be recurrences given that the current state is a recurrence; here, by a recurrence we mean that the distance of a state from some previous one is smaller than or equal to the precision $\varepsilon$. To be more precise, take an ergodic measure $\mu$ of the system $(X, f)$. Then, by [9, for $\mu$-a.e. $x \in X$ and for all but countably many $\varepsilon>0$, asymptotic correlation sum exists and is equal to the correlation integral of $\mu$

$$
c_{m}(\mu, \varepsilon)=\mu \times \mu\left\{(y, z) \in X \times X: \varrho_{m}(y, z) \leq \varepsilon\right\} .
$$

Thus if $Y, Z$ are independent $X$-valued random variables with distribution $\mu$, then asymptotic correlation sum $c_{m}(x, \varepsilon)$ is (typically) equal to the probability that $f^{i}(Y)$ and $f^{i}(Z)$ are $\varepsilon$-close for every $i \in[0, m)$. Consequently, asymptotic recurrence determinism $\operatorname{rdet}_{m}(x, \varepsilon)$ is (typically) equal to the conditional probability that $f^{i}(Y)$ and $f^{i}(Z)$ are $\varepsilon$-close for every $i \in[0, m)$, given that $Y$ and $Z$ are $\varepsilon$-close. For more details see [7]. 
Asymptotic correlation sum and asymptotic recurrence determinism do not depend on the beginning of the trajectory, as is stated in the next lemma.

Lemma 11. Let $(X, f)$ be a dynamical system, $x \in X, h \in \mathbb{N}, m \in \mathbb{N} \cup\{\infty\}$, and $\varepsilon>0$. Then

$$
\bar{c}_{m}\left(f^{h}(x), \varepsilon\right)=\bar{c}_{m}(x, \varepsilon), \quad \underline{c}_{m}\left(f^{h}(x), \varepsilon\right)=\underline{c}_{m}(x, \varepsilon),
$$

and

$$
\overline{\operatorname{rdet}}_{m}\left(f^{h}(x), \varepsilon\right)=\overline{\operatorname{rdet}}_{m}(x, \varepsilon), \quad \underline{\operatorname{rdet}}_{m}\left(f^{h}(x), \varepsilon\right)=\underline{\operatorname{rdet}}_{m}(x, \varepsilon) .
$$

Proof. It suffices to apply the following inequalities for correlation sums, valid for every integer $n$ :

$$
\left(\frac{n+h}{n}\right)^{2} C_{m}(x, n+h, \varepsilon)-\frac{2 h n+h^{2}}{n^{2}} \leq C_{m}\left(f^{h}(x), n, \varepsilon\right) \leq\left(\frac{n+h}{n}\right)^{2} C_{m}(x, n+h, \varepsilon) .
$$

The following result easily follows from Lemma 8 and from basic properties of lim inf and lim sup.

Lemma 12. Let $(X, f)$ be a dynamical system, $x \in X, m \in \mathbb{N} \cup\{\infty\}$, and $\varepsilon>0$. Then $\underline{c}_{1}(x, \varepsilon)>0$ and

$$
\frac{\underline{c}_{m}(x, \varepsilon)}{\bar{c}_{1}(x, \varepsilon)} \leq \underline{\operatorname{rdet}}_{m}(x, \varepsilon) \leq \overline{\operatorname{rdet}}_{m}(x, \varepsilon) \leq \frac{\bar{c}_{m}(x, \varepsilon)}{\underline{c}_{1}(x, \varepsilon)} .
$$

4. Proofs of Theorems 1 and 2

Here we give proofs of Theorems 1 and 2 . In Section 4.1 we deal with the simplest case of finite $\omega$-limit sets. Some simple lemmas regarding solenoidal $\omega$-limit sets are given in Section 4.2. The case when an $\omega$-limit set contains non-separable points is described in Section 4.3 Finally, in Sections 4.4 and 4.5 we deal with the remaining case of solenoidal $\omega$-limit sets without non-separable points.

\subsection{Finite $\omega$-limit sets.}

Proposition 13. Let $(X, f)$ be a dynamical system and $x \in X$ have $\omega_{f}(x)$ of finite cardinality $p$. Then, for every sufficiently small $\varepsilon>0$ and every $m \in \mathbb{N} \cup\{\infty\}$,

$$
c_{m}(x, \varepsilon)=\frac{1}{p} \quad \text { and } \quad \operatorname{rdet}_{m}(x, \varepsilon)=1 .
$$

Proof. We may assume that $m$ is finite. Write $x_{i}=f^{i}(x)$ for $i \geq 0$ and $\omega_{f}(x)=\left\{y_{0}, y_{1}, \ldots, y_{p-1}\right\}$, where $f^{k}\left(y_{0}\right)=y_{k \bmod p}$ for every $k \geq 0$. Take arbitrary $\varepsilon>0$ such that $\varrho\left(y_{k}, y_{l}\right) \geq 2 \varepsilon$ for every $k \neq l$. Since $x$ is attracted by the $p$-cycle $\left(y_{0}, y_{1}, \ldots, y_{p-1}\right)$, there is $i_{0}$ such that $\varrho\left(x_{i_{0}+i}, y_{i \bmod p}\right)<\varepsilon / 2$ for every $i \geq 0$. By Lemma 11 we may assume that $i_{0}=0$. Hence, for $i, j \geq 0$ with $i \equiv j(\bmod p)$ we have

$$
\varrho\left(x_{i}, x_{j}\right) \leq \varrho\left(x_{i}, y_{i \bmod p}\right)+\varrho\left(y_{i \bmod p}, y_{j \bmod p}\right)+\varrho\left(y_{j \bmod p}, x_{j}\right)<\varepsilon,
$$

and for $i, j \geq 0$ with $i \not \equiv j(\bmod p)$ we have

$$
\varrho\left(x_{i}, x_{j}\right) \geq \varrho\left(y_{i \bmod p}, y_{j \bmod p}\right)-\varrho\left(x_{i}, y_{i \bmod p}\right)-\varrho\left(y_{j \bmod p}, x_{j}\right)>2 \varepsilon-\varepsilon=\varepsilon .
$$

That is, for any $i, j \geq 0, \varrho_{m}\left(x_{i}, x_{j}\right) \leq \varepsilon$ if and only $i \equiv j(\bmod p)$. Now the assertion easily follows.

4.2. Solenoidal $\omega$-limit sets - basic facts. Till the end of Section 4 we will use the notation from Section 2.2. Fix a map $f: I \rightarrow I$ and a point $x \in I$, and put $x_{i}=f^{i}(x)$ for every $i \geq 0$. Take $m \in \mathbb{N} \cup\{\infty\}$ and $t \geq 0$. For every $a, b \in \mathcal{A}^{t}$ put

$$
\operatorname{dist}_{m}\left(K_{a}, K_{b}\right)=\max _{i \in[0, m)} \operatorname{dist}\left(K_{a+i}, K_{b+i}\right), \quad \operatorname{diam}_{m}\left(K_{a}, K_{b}\right)=\max _{i \in[0, m)} \operatorname{diam}\left(K_{a+i} \cup K_{b+i}\right) .
$$

Note that, due to periodicity of intervals $K_{a}$, $\operatorname{dist}_{m}=\operatorname{dist}_{p_{t}}$ and $\operatorname{diam}_{m}=\operatorname{diam}_{p_{t}}$ for every $m \geq p_{t}$. For $\varepsilon>0$ define

$$
\begin{aligned}
& N_{m}(x, t, \varepsilon)=\#\left\{(a, b) \in \mathcal{A}^{t} \times \mathcal{A}^{t}: \operatorname{dist}_{m}\left(K_{a}, K_{b}\right)<\varepsilon\right\} \\
& N_{m}^{\circ}(x, t, \varepsilon)=\#\left\{(a, b) \in \mathcal{A}^{t} \times \mathcal{A}^{t}: \operatorname{diam}_{m}\left(K_{a}, K_{b}\right) \leq \varepsilon\right\} .
\end{aligned}
$$

Lemma 14. Let $f: I \rightarrow I$ be continuous and $x \in I$ be such that $\omega_{f}(x)$ is solenoidal. Then, for every $m \in \mathbb{N} \cup\{\infty\}, t \geq 0$, and $\varepsilon>0$, 
(1) $N_{m}(x, t, \varepsilon) \geq N_{m+1}(x, t, \varepsilon)$ and $N_{m}^{\circ}(x, t, \varepsilon) \geq N_{m+1}^{\circ}(x, t, \varepsilon)$;

(2) $N_{m}(x, t, \varepsilon)=N_{p_{t}}(x, t, \varepsilon)$ and $N_{m}^{\circ}(x, t, \varepsilon)=N_{p_{t}}^{\circ}(x, t, \varepsilon)$ if $m \geq p_{t}$;

(3) $N_{m}^{\circ}(x, t, \varepsilon) \leq N_{m}(x, t, \varepsilon)$;

(4) $N_{m}^{\circ}(x, t, \varepsilon) \cdot p_{t}^{-2} \leq \underline{c}_{m}(x, \varepsilon) \leq \bar{c}_{m}(x, \varepsilon) \leq N_{m}(x, t, \varepsilon) \cdot p_{t}^{-2}$;

(5) if $N_{1}^{\circ}(x, t, \varepsilon)>0$ then

$$
\frac{N_{m}^{\circ}(x, t, \varepsilon)}{N_{1}(x, t, \varepsilon)} \leq \underline{\operatorname{rdet}}_{m}(x, \varepsilon) \leq \overline{\operatorname{rdet}}_{m}(x, \varepsilon) \leq \frac{N_{m}(x, t, \varepsilon)}{N_{1}^{\circ}(x, t, \varepsilon)} .
$$

Proof. Properties (10), (2) are trivial and (3) follows from the fact that every $K_{a}\left(a \in \mathcal{A}^{t}\right)$ is non-degenerate. To prove (44), by Lemma 11 and the fact that $\omega_{f}(x)$ is infinite we may assume that $x \in K_{0^{t}}$ and that the orbit of $x$ does not intersect the boundary of any $K_{a}\left(a \in \mathcal{A}^{t}\right)$. Thus $x_{i} \in K_{0^{t}+i}$ for every $i$, and

$$
\operatorname{dist}_{m}\left(K_{0^{t}+i}, K_{0^{t}+j}\right)<\varrho_{m}\left(x_{i}, x_{j}\right)<\operatorname{diam}_{m}\left(K_{0^{t}+i}, K_{0^{t}+j}\right) \quad \text { for every } i \neq j .
$$

Since intervals $K_{a}\left(a \in \mathcal{A}^{t}\right)$ are $p_{t}$-periodic, we have

$$
\frac{k_{n}^{2}}{\left(k_{n}+1\right)^{2} \cdot p_{t}^{2}} \cdot N_{m}^{\circ}(x, t, \varepsilon)<C_{m}(x, n, \varepsilon)<\frac{\left(k_{n}+1\right)^{2}}{k_{n}^{2} \cdot p_{t}^{2}} \cdot N_{m}(x, t, \varepsilon)
$$

for every $n \geq p_{t}$, where $k_{n}=\left\lfloor n / p_{t}\right\rfloor$. A passage to the limit $n \rightarrow \infty$ gives (4).

Since (5) follows from (4) and Lemma 12, the proof is finished.

The following proposition states that, for zero entropy interval maps, asymptotic correlation sum $c_{\infty}$ can distinguish points with finite $\omega$-limit set from those with infinite one.

Proposition 15. Let $f: I \rightarrow I$ be a continuous map with zero entropy and $x \in I$. Then the following conditions are equivalent:

(1) $\omega_{f}(x)$ is finite;

(2) there is $p \in \mathbb{N}$ such that $c_{\infty}(x, \varepsilon)=1 / p$ for every sufficiently small $\varepsilon>0$;

(3) $\liminf _{\varepsilon \rightarrow 0} \underline{c}_{\infty}(x, \varepsilon)>0$;

(4) $\lim \sup _{\varepsilon \rightarrow 0} \bar{c}_{\infty}(x, \varepsilon)>0$.

Proof. The fact that (1) implies (2) was proved in Proposition [13, and (21) $\Longrightarrow$ (3) $\Longrightarrow$ (4) is obvious. To prove (41) $\Longrightarrow$ (11) assume that $\omega_{f}(x)$ is infinite, hence solenoidal with $p_{t}=2^{t}$ by Lemma 6] Take any $t \in \mathbb{N}$ and $0<\varepsilon \leq \min \left\{\operatorname{dist}\left(K_{a}, K_{b}\right): a, b \in \mathcal{A}^{t}, a \neq b\right\}$. Then $\bar{c}_{\infty}(x, \varepsilon) \leq 2^{-t}$ by Lemma 14(4). Since $t$ is arbitrary, we have $\lim _{\varepsilon} \bar{c}_{\infty}(x, \varepsilon)=0$.

4.3. Solenoidal $\omega$-limit sets with non-separable points. The following is related to [13, Lemma 4.1].

Lemma 16. Let $f: I \rightarrow I$ be continuous and $x \in I$ be such that $\omega_{f}(x)$ is solenoidal. Let $\alpha \in \Sigma$ be such that $K_{\alpha}=\left[y_{\alpha}, z_{\alpha}\right]$ is non-degenerate and both endpoints $y_{\alpha}, z_{\alpha}$ belong to $\omega_{f}(x)$. Then for every $s \in \mathbb{N}$ there are $t=t^{(s)}>s$ and $b=b^{(s)}, c=c^{(s)} \in \mathcal{A}^{t}$ such that

$$
b_{[0, s)}=c_{[0, s)}=\alpha_{[0, s)} \quad \text { and } \quad K_{b}<K_{\alpha[0, t)}<K_{c} .
$$

Moreover, $t^{(s)}<t^{(s+1)}$ for every $s$.

Proof. Put $t^{(0)}=0$. Fix an integer $s \geq 1$ and assume that $t^{(s-1)}$ has been defined. Since $\omega_{f}(x)$ is infinite, $y_{\alpha}$ and $z_{\alpha}$ are not isolated points of $\omega_{f}(x)$. Moreover, the interior $\left(y_{\alpha}, z_{\alpha}\right)$ of $K_{\alpha}$ is wandering [1, Corollary 3.2(2)]. Thus there are increasing sequences $\left(n_{k}\right)_{k}$ and $\left(m_{k}\right)_{k}$ of integers such that $f^{n_{k}}(x) \nearrow y_{\alpha}$ and $f^{m_{k}}(x) \searrow z_{\alpha}$; we may assume that $f^{n_{k}}(x), f^{m_{k}}(x) \in Q_{s}$ for all sufficiently large $k$. This, together with $y_{\alpha}, z_{\alpha} \in K_{\alpha[0, s)}$, implies that there is $k_{0}$ such that $f^{n_{k}}(x), f^{m_{k}}(x) \in K_{\alpha[0, s)}$ for every $k \geq k_{0}$.

Fix any $k \geq k_{0}$. Since $f^{n_{k}}(x)<y_{\alpha}$, there is $t_{0}>s$ such that $f^{n_{k}}(x) \in Q_{t_{0}} \backslash K_{\alpha\left[0, t_{0}\right)}$. Hence there is $b^{\prime} \in \mathcal{A}^{t_{0}}$ with $f^{n_{k}}(x) \in K_{b^{\prime}}<K_{\alpha\left[0, t_{0}\right)}$; moreover, by the choice of $k_{0}, b_{[0, s)}^{\prime}=\alpha_{[0, s)}$. Analogously, there are $t_{1}>s$ and $c^{\prime} \in \mathcal{A}^{t_{1}}$ such that $f^{m_{k}}(x) \in K_{c^{\prime}}>K_{\alpha\left[0, t_{0}\right)}$ and $c_{[0, s)}^{\prime}=\alpha_{[0, s)}$. Now it suffices to put $t=t^{(s)}=\max \left\{t_{0}, t_{1}, t^{(s-1)}+1\right\}$ and take arbitrary $b, c \in \mathcal{A}^{t}$ with $b_{\left[0, t_{0}\right)}=b^{\prime}$ and $c_{\left[0, t_{1}\right)}=c^{\prime}$.

Lemma 17. Let $f: I \rightarrow I$ be continuous and $x \in I$ be such that $\omega_{f}(x)$ is solenoidal. Assume that $\alpha \in \Sigma$ and $\varepsilon>0$ are such that diam $K_{\alpha} \geq \varepsilon$ and $\partial K_{\alpha} \subseteq \omega_{f}(x)$. Then

$$
c_{\infty}(x, \varepsilon)=0 \quad \text { and } \quad \operatorname{rdet}_{\infty}(x, \varepsilon)=0 .
$$


Proof. Fix arbitrary $s \geq 1$ and $h \in \mathbb{N} \backslash p_{s} \mathbb{N}$. Let $t=t^{(s)}, b=b^{(s)}$, and $c=c^{(s)}$ be as in Lemma 16. By Lemma [11 we may assume that $x \in K_{0^{t}}$. Let $j_{0}$ be such that $0^{s}+j_{0}=\alpha_{[0, s)}$. Put $u=0^{s}+h+j_{0}$; then $u \neq \alpha_{[0, s)}$ by the choice of $h$.

Assume first that $K_{u}<K_{\alpha[0, s)}$. Let $j_{1} \in\left[0, p_{t}\right)$ be such that $0^{t}+j_{0}+j_{1}=c$. Since $c_{[0, s)}=\alpha_{[0, s)}$ and $0^{t}+j_{0}$ starts with $\alpha_{[0, s)}, j_{1}$ is a multiple of $p_{s}$. Further, $\left(0^{t}+h+j_{0}+j_{1}\right)_{[0, s)}=0^{s}+h+j_{0}=u$. Thus $x_{j_{0}+j_{1}+l p_{t}} \in K_{c}$ and $x_{h+j_{0}+j_{1}+l p_{t}} \in K_{u}$ for every $l \geq 0$. Since $K_{u}<\min K_{\alpha[0, s)} \leq K_{\alpha[0, t)}<K_{c}$, we conclude that

$$
\varrho_{\infty}\left(x_{i}, x_{h+i}\right) \geq \varrho_{p_{t}}\left(x_{i}, x_{h+i}\right)>\operatorname{diam} K_{\alpha[0, t)}>\operatorname{diam} K_{\alpha} \geq \varepsilon \quad \text { for every } i \geq 0 .
$$

The same conclusion can be analogously obtained in the case when $K_{u}>K_{\alpha[0, s)}$; one only needs to replace $c$ with $b$. Thus in both cases we have $\varrho_{\infty}\left(x_{i}, x_{h+i}\right)>\varepsilon$ for every $i \geq 0$ and every $h \in \mathbb{N} \backslash p_{s} \mathbb{N}$. This immediately implies that $\bar{c}_{\infty}(x, \varepsilon) \leq\left(1 / p_{s}\right)$. Since $s$ is arbitrary and $\lim _{s} p_{s}=\infty, c_{\infty}(x, \varepsilon)=0$ and hence, by Lemma 12, $\operatorname{rdet}_{\infty}(x, \varepsilon)=0$.

Proposition 18. Let $f: I \rightarrow I$ be continuous and $x \in I$. If $\omega_{f}(x)$ is a solenoidal $\omega$-limit set containing two non-separable points $y$ and $z$, then

$$
c_{\infty}(x, \varepsilon)=\operatorname{rdet}_{\infty}(x, \varepsilon)=0 \quad \text { for every } \varepsilon \leq \varrho(y, z) .
$$

Proof. The assertion follows immediately from Lemmas 17 and 7.

In the proof of Theorem 1 we will need the following lemma.

Lemma 19. Let $f: I \rightarrow I$ have zero entropy and $x \in I$ be such that $\omega_{f}(x)$ is solenoidal and contains non-separable points $y, z$. Let $C$ be the set from Lemma 4. Then $y, z \in C$.

Proof. By Lemma 7 we may assume that $y=y_{\alpha}$ and $z=z_{\alpha}$ for some $\alpha \in \Sigma$. Lemma 16 asserts that any open interval $J \ni y$ contains a (periodic) interval $K_{b} \not \supset y$. Hence $y$ is not an isolated point of $Q \cap \Omega(f)$ and so it belongs to $C$ (recall that $C$ is the set of all limit points of $Q \cap \Omega(f)$ ). Analogously we can prove that $z \in C$.

\subsection{Solenoidal $\omega$-limit sets without non-separable points - the first part.}

Proposition 20. Let $f: I \rightarrow I$ be continuous. Let $x \in I$ be such that $\omega_{f}(x)$ is solenoidal and does not contain non-separable points. Then

$$
\underline{\operatorname{rdet}}_{\infty}(x, \varepsilon) \geq \underline{c}_{\infty}(x, \varepsilon)>0 \quad \text { for every } \varepsilon>0 .
$$

Proof. Fix any $\varepsilon>0$. Let $\left\{\alpha^{(1)}, \ldots, \alpha^{(m)}\right\}$ be the set of all $\alpha \in \Sigma$ with $\operatorname{diam} K_{\alpha} \geq(\varepsilon / 2)$. Since interiors of $K_{\alpha^{(j)}}$ are wandering [1, Corollary 3.2(2)] and $x$ is not eventually periodic, the orbit of $x$ visits $\bigcup_{j} K_{\alpha^{(j)}}$ at most finitely many times; by Lemma 11 we may assume that

$$
K_{\alpha^{(j)}} \cap \operatorname{Orb}_{f}(x)=\emptyset \quad \text { for every } j \in[1, m] .
$$

Fix any $j \in[1, m]$. Since $\omega_{f}(x)$ contains no non-separable points,

$$
K_{\alpha^{(j)}} \cap \omega_{f}(x) \text { is a singleton subset of } \partial K_{\alpha^{(j)}}=\left\{y_{\alpha^{(j)}}, z_{\alpha^{(j)}}\right\}
$$

by Lemmas 4 and 7 Thus there is $s_{j} \in \mathbb{N}$ with the following properties: if $y_{\alpha^{(j)}} \in \omega_{f}(x)$ then

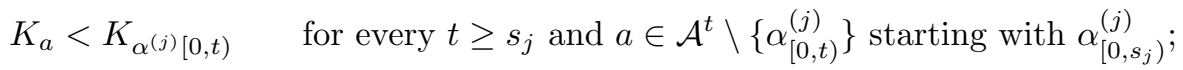

and if $z_{\alpha^{(j)}} \in \omega_{f}(x)$ then

$$
K_{a}>K_{\alpha^{(j)}[0, t)} \quad \text { for every } t \geq s_{j} \text { and } a \in \mathcal{A}^{t} \backslash\left\{\alpha_{[0, t)}^{(j)}\right\} \text { starting with } \alpha_{\left[0, s_{j}\right)}^{(j)} .
$$

To see this it suffices to realize that if (4.3) is not true then $z_{\alpha^{(j)}} \in \omega_{f}(x)$, and if (4.4) is not true then $y_{\alpha}(j) \in \omega_{f}(x)$.

We claim that there exists $s \geq \max \left\{s_{j}: j \in[1, m]\right\}$ such that, for every $b \in \mathcal{A}^{s}$,

$$
\text { either } b=\alpha_{[0, s)}^{(j)} \text { and } \lambda\left(K_{b} \backslash K_{\alpha^{(j)}}\right)<\varepsilon \text { for some } j, \quad \text { or } \operatorname{diam} K_{b}<\varepsilon \text {. }
$$

To prove it suppose that there are a sequence $\left(s_{l}^{\prime}\right)_{l} \nearrow \infty$ of integers and a sequence $\left(b^{(l)}\right)_{l}$ of words $b^{(l)} \in \mathcal{A}^{s_{l}^{\prime}}$, such that diam $K_{b^{(l)}} \geq \varepsilon$ and $b^{(l)}$ is not a beginning of any $\alpha^{(j)}$. Denote by $w_{l}$ the middle of $K_{b^{(l)}}$; without 
loss of generality we may assume that $\left(w_{l}\right)_{l}$ converges to some $w \in I$. Since $w_{l} \pm(\varepsilon / 2) \in K_{b^{(l)}}$, we have that $L=[w-\varepsilon / 4, w+\varepsilon / 4]$ is a subset of $K_{b^{(l)}}$ for every sufficiently large $l$. Hence $L \subseteq Q$ and so, due to $\operatorname{diam} L=\varepsilon / 2, L$ is a subset of some $K_{\alpha^{(j)}}$. But then $K_{\alpha^{(j)}} \subseteq K_{b^{(l)}}$, that is, $b^{(l)}$ is a beginning of $\alpha^{(j)}$, a contradiction. Thus we have proved that there is arbitrarily large $s$ such that, for every $b \in \mathcal{A}^{s}$, either $b=\alpha_{[0, s)}^{(j)}$ or diam $K_{b}<\varepsilon$. Since $K_{\alpha^{(j)}}=\bigcap_{s} K_{\alpha^{(j)}[0, s)}$, we see that for $s$ large enough we also have $\lambda\left(K_{b} \backslash K_{\alpha^{(j)}}\right)<\varepsilon$. Hence (4.5) is proved.

We may assume that $x \in Q_{s}$. Take any $h \in p_{s} \mathbb{N}$. Since $h$ is a multiple of $p_{s}$, for every $i$ there is $b^{(i)} \in \mathcal{A}^{s}$ with $x_{i}, x_{i+h} \in K_{b^{(i)}}$. We claim that

$$
\left|x_{i+h}-x_{i}\right| \leq \varepsilon \quad \text { for every } i
$$

To prove it, fix any $i$ and put $b=b^{(i)}$. If $b \neq \alpha_{[0, s)}^{(j)}$ for every $j$, then $\operatorname{diam} K_{b}<\varepsilon$ by (4.5), and (4.6) is immediate. So assume that $b=\alpha_{[0, s)}^{(j)}$ for some $j$; put $\alpha=\alpha^{(j)}$. By (4.2), either $y_{\alpha} \in \omega_{f}(x)$ or $z_{\alpha} \in \omega_{f}(x)$; we will consider only the former case, the latter one being similar. Since $x_{i} \notin K_{\alpha}$ by (4.1), there are $t>s$ and $c \in \mathcal{A}^{t}$ starting with $b$ such that $x_{i} \in K_{c}$ and $c \neq \alpha_{[0, t)}$. Thus $K_{c}<K_{\alpha[0, t)}$ by (4.3), and

$$
x_{i} \in K_{c}<\min K_{\alpha[0, t)} \leq \min K_{\alpha}=y_{\alpha} .
$$

Analogously, $x_{i+h}<y_{\alpha}$. Thus, by (4.5),

$$
\left|x_{i+h}-x_{i}\right| \leq \operatorname{diam}\left[y_{b}, y_{\alpha}\right) \leq \lambda\left(K_{b} \backslash K_{\alpha}\right)<\varepsilon .
$$

Hence (4.6) is proved.

Since (4.6) is true for every $h \in p_{s} \mathbb{N}$, we have $\underline{c}_{\infty}(x, \varepsilon) \geq\left(1 / p_{s}\right)>0$. The proposition is proved.

4.5. Solenoidal $\omega$-limit sets without non-separable points - the second part. In this section we assume that $p_{t}=2^{t}$ for every $t \geq 1$; so $\mathcal{A}^{t}=\{0,1\}^{t}$ and $\Sigma=\{0,1\}^{\mathbb{N}_{0}}$. Fix $\varepsilon>0$ and for every $t \geq 0$ put

$$
\mathcal{L}_{t}(\varepsilon)=\left\{a \in \mathcal{A}^{t}: \operatorname{diam}\left(K_{a}\right) \geq \varepsilon\right\} \quad \text { and } \quad \mathcal{L}_{\infty}(\varepsilon)=\left\{\alpha \in \Sigma: \operatorname{diam}\left(K_{\alpha}\right) \geq \varepsilon\right\}
$$

denote the cardinalities of these (finite) sets by $\ell_{t}(\varepsilon)$ and $\ell_{\infty}(\varepsilon)$, respectively.

Lemma 21. Fix any $\varepsilon>0$. Then

(1) $\left(\ell_{t}(\varepsilon) \cdot 2^{-t}\right)_{t}$ is non-increasing;

(2) $\left(\lambda_{t}(\varepsilon)\right)_{t}$ is strictly decreasing, where $\lambda_{t}(\varepsilon)=\sum_{a \in \mathcal{L}_{t}(\varepsilon)} \operatorname{diam}\left(K_{a}\right)$;

(3) $\ell_{t}(\varepsilon)=\ell_{\infty}(\varepsilon)$ for every sufficiently large $t$.

Proof. If $a \notin \mathcal{L}_{t}$, then trivially $[a] \cap \mathcal{L}_{t^{\prime}}=\emptyset$ for every $t^{\prime} \geq t$. So $\ell_{t^{\prime}} \leq \ell_{t} \cdot 2^{t^{\prime}-t}$ and (1) is proved.

Since (2) is trivial, it suffices to prove (3). For every $t$ define $\varphi_{t}: \mathcal{L}_{t+1} \rightarrow \mathcal{L}_{t}$ by $\varphi_{t}(a)=a_{[0, t)}$; since $K_{a} \subseteq K_{\varphi_{t}(a)}, \varphi_{t}(a) \in \mathcal{L}_{t}$ for every $a \in \mathcal{L}_{t+1}$. If $\varphi_{t}$ is not surjective, then $\lambda_{t+1} \leq \lambda_{t}-\varepsilon$. Combined with (2) this implies that $\varphi_{t}$ is surjective for all but finitely many $t$. Hence, for some $s$,

$$
\ell_{s} \leq \ell_{s+1} \leq \ell_{s+2} \leq \ldots
$$

Since $\ell_{t} \leq(1 / \varepsilon)$ for every $t$, by (4.7) there is an integer $k$ such that $\ell_{t}=k$ for every sufficiently large $t$. Now trivially $\ell_{\infty}=k$.

For any $t \geq 0$ and $a \in \mathcal{A}^{t}$, denote the words $a 00, a 01, a 10, a 11$ by $a_{*}, a_{\circ}, a^{\circ}, a^{*}$ in such a way that

$$
K_{a_{*}}<K_{a_{\circ}}<K_{a^{\circ}}<K_{a^{*}} .
$$

Since either $K_{a_{*}} \cup K_{a_{\circ}} \subseteq K_{a 0}$ and $K_{a^{\circ}} \cup K_{a^{*}} \subseteq K_{a 1}$, or vice versa, we have

$$
a_{* t}=a_{\circ t} \neq a_{t}^{\circ}=a_{t}^{*} \text {. }
$$

Put

$$
\varepsilon_{t}=\max _{a \in \mathcal{A}^{t}} \operatorname{dist}\left(K_{a_{*}}, K_{a^{*}}\right)
$$

and note that $\varepsilon_{t}>\operatorname{diam}\left(K_{a_{\circ}} \cup K_{a^{\circ}}\right)$ for every $a \in \mathcal{A}^{t}$.

Lemma 22. Let $f: I \rightarrow I$ be continuous. Let $x \in I$ be such that $\omega_{f}(x)$ is solenoidal and does not contain non-separable points. Then

$$
\lim _{t \rightarrow \infty} \varepsilon_{t}=0
$$


Proof. By Lemma 21(3) there is $s$ such that, for every $a \in \mathcal{A}^{*}$ with $|a| \geq s$,

$$
\operatorname{diam}\left(K_{a}\right) \geq \varepsilon \quad \text { if and only if } \quad \text { there is } \alpha \in \Sigma \cap[a] \text { with } \operatorname{diam}\left(K_{\alpha}\right) \geq \varepsilon \text {. }
$$

Suppose that $\lim \sup _{t} \varepsilon_{t}>0$; that is, there are $\bar{\varepsilon}>0$, integers $s \leq t_{1}<t_{2}<\ldots$, and words $a^{n} \in \mathcal{A}^{t_{n}}$ $(n \in \mathbb{N})$, such that $\operatorname{diam}\left(K_{a^{n}}\right)>\operatorname{dist}\left(K_{a_{*}^{n}}, K_{a^{n *}}\right)>\bar{\varepsilon}$ for every $n$. By going to a subsequence if necessary, we may assume that there is $\alpha \in \Sigma$ such that $K_{\alpha}=\bigcap_{n} K_{a^{n}}$. By the choice of $\bar{\varepsilon}, K_{a_{*}^{n}}<K_{\alpha}<K_{a^{n *}}$ for every sufficiently large $n$, and so both end points $y_{\alpha}, z_{\alpha}$ of $K_{\alpha}$ belong to $\omega_{f}(x)$. Hence, by Lemma 7, $\omega_{f}(x)$ contains non-separable points.

Lemma 23. Let $s \geq 0, t \geq s+2, a \in \mathcal{A}^{s}$, and $b \in \mathcal{A}^{t} \cap[a]$. Let $h$ be an odd multiple of $2^{s}$. Then for every $u, v \in\{0,1\}$ there is a unique integer $i \in[0,3]$ such that either $b+i 2^{s} \in[a 0 u]$ and $b+h+i 2^{s} \in[a 1 v]$, or $b+i 2^{s} \in[a 1 v]$ and $b+h+i 2^{s} \in[a 0 u]$.

Proof. We may assume that $s=0, t=2, h \in\{1,3\}$, and $b=00$. In such a case the statement of the lemma can be easily verified.

Lemma 24. Let $t \geq 2$ and $h$ be an odd multiple of $2^{s}$ for some $s \in[0, t-2]$. Then

$$
\operatorname{dist}_{\infty}\left(K_{b}, K_{b+h}\right) \geq \varepsilon_{s} \quad \text { for every } b \in \mathcal{A}^{t} .
$$

Proof. Let $a \in \mathcal{A}^{s}$ be such that $\varepsilon_{s}=\operatorname{dist}\left(K_{a_{*}}, K_{a^{*}}\right)$. Fix arbitrary $b \in \mathcal{A}^{t}$. Since $\operatorname{dist}_{\infty}\left(K_{b}, K_{c}\right)=$ $\operatorname{dist}_{\infty}\left(K_{b+j}, K_{c+j}\right)$ for every $c$ and $j$, we may assume that $b \in[a]$. By (4.8) and Lemma 23, there is $i \in[0,3]$ such that either $b+i 2^{s} \in\left[a_{*}\right]$ and $b+h+i 2^{s} \in\left[a^{*}\right]$, or vice versa. In both cases,

$$
\operatorname{dist}_{\infty}\left(K_{b}, K_{b+h}\right) \geq \operatorname{dist}\left(K_{b+i 2^{s}}, K_{b+h+i 2^{s}}\right) \geq \operatorname{dist}\left(K_{a_{*}}, K_{a^{*}}\right)=\varepsilon_{s} .
$$

The next lemma follows immediately from Lemma 24

Lemma 25. Let $t \geq 2$ and $h$ be an integer which is not a multiple of $2^{t-1}$. Then, for every $b \in \mathcal{A}^{t}$,

$$
\operatorname{dist}_{\infty}\left(K_{b}, K_{b+h}\right) \geq \min \left\{\varepsilon_{0}, \ldots, \varepsilon_{t-2}\right\} .
$$

Lemma 26. Let $t \geq 2$ be such that $\varepsilon_{t-2}=\min \left\{\varepsilon_{0}, \ldots, \varepsilon_{t-2}\right\}$. Then

$$
\overline{\operatorname{rdet}}_{\infty}\left(x, \varepsilon_{t-2}\right) \leq \frac{4}{5} \text {. }
$$

Proof. For abbreviation, write $\varepsilon, N_{m}, N_{m}^{\circ}$ instead of $\varepsilon_{t-2}, N_{m}\left(x, t, \varepsilon_{t-2}\right), N_{m}^{\circ}\left(x, t, \varepsilon_{t-2}\right)$, respectively. For $m \in\{1, \infty\}$ and $h \in\left[0,2^{t}\right)$ put $n_{m}(h)=\#\left\{b \in \mathcal{A}^{t}: \operatorname{dist}_{m}\left(K_{b}, K_{b+h}\right)<\varepsilon\right\}$ and $n_{m}^{\circ}(h)=\#\left\{b \in \mathcal{A}^{t}\right.$ : $\left.\operatorname{diam}_{m}\left(K_{b}, K_{b+h}\right) \leq \varepsilon\right\}$; note that $N_{m}=\sum_{h} n_{m}(h)$ and $N_{m}^{\circ}=\sum_{h} n_{m}^{\circ}(h)$. By Lemma [25] $n_{\infty}(h)=0$ for every $h \notin\left\{0,2^{t-1}\right\}$; thus

$$
N_{\infty} \leq 2 \cdot 2^{t}
$$

On the other hand, if $h=2^{t-2}$ or $h=3 \cdot 2^{t-2}$ then $n_{1}^{\circ}(h) \geq(1 / 4) \cdot 2^{t}$; to see this, use (4.8), Lemma 23, and the fact that $\operatorname{diam}\left(K_{a_{\circ}} \cup K_{a^{\circ}}\right)<\operatorname{dist}\left(K_{a_{*}}, K_{a^{*}}\right) \leq \varepsilon$ for every $a \in \mathcal{A}^{t-2}$. Hence

$$
N_{1}^{\circ}-N_{\infty} \geq 2 \cdot \frac{2^{t}}{4}=2^{t-1} .
$$

Applying Lemma 14(5) and inequalities (4.9), (4.10) we conclude that

$$
\overline{\operatorname{rdet}}_{\infty}(x, \varepsilon) \leq \frac{N_{\infty}}{N_{1}^{\circ}} \leq \frac{N_{\infty}}{N_{\infty}+2^{t-1}} \leq \frac{1}{1+\frac{1}{4}}=\frac{4}{5} .
$$

From Lemmas 26 and 22 we immediately obtain the following proposition stating that, for solenoidal $\omega$-limit sets, we cannot have recurrence determinism converging to one as $\varepsilon \rightarrow 0$. It is interesting that we even have an upper bound for limes inferior depending neither on $f$ nor on $x$.

Proposition 27. Let $f: I \rightarrow I$ be continuous and $x \in I$ be such that $\omega_{f}(x)$ is solenoidal and does not contain non-separable points. Then

$$
\liminf _{\varepsilon \rightarrow 0} \overline{\operatorname{rdet}}_{\infty}(x, \varepsilon) \leq \frac{4}{5}
$$




\subsection{Proofs of Theorems 1 and 2 ,}

Proof of Theorem 2. It suffices to prove implications from left to right. Propositions [13 and 18 prove (1) and (3) of Theorem 2. Assertion (2) is shown in Propositions 20] and 27.

Proof of Theorem [1. We start by proving implications from left to right. The implication from (1) follows immediately from Proposition [13. If $f$ is not Li-Yorke chaotic then the $\omega$-limit sets of $f$ are either finite, or solenoidal without non-separable points [13. Theorem 2.2]. Thus Propositions 13 and 20 show the implication from (2).

Assume now that $f$ is Li-Yorke chaotic. If the topological entropy of $f$ is zero then, by [13, Theorem 2.2], there is $x \in I$ such that $\omega_{f}(x)$ contains non-separable points. Let $C$ be the (Cantor) set from Lemma 4 . Then $\omega_{f}(y)=C$ for every $y \in C$. Since $C$ contains non-separable points $z$ and $z^{\prime}$ by Lemma 19, Proposition 18 implies that $\operatorname{rdet}_{\infty}(y, \varepsilon)=0$ for every $y \in C$ and every $\varepsilon \leq \varrho\left(z, z^{\prime}\right)$. If the topological entropy of $f$ is positive, then [14, Theorem B] asserts that there is a Cantor set $C \subseteq I$ such that $\left(\bar{c}_{m}(x, \varepsilon)\right)_{m}$ decreases to zero exponentially fast (and hence $\operatorname{rdet}_{\infty}(x, \varepsilon)=c_{\infty}(x, \varepsilon)=0$ ) for every $x \in C$ and every sufficiently small $\varepsilon>0$. Thus also the implication from (3) is proved.

Now we prove implications from right to left. Since the conditions on recurrence determinism from (2) and (3) are mutually exclusive, the equivalences from (2) and (3) are immediate. Assume now that $\operatorname{rdet}_{\infty}(x, \varepsilon)=1$ for every $x \in I$ and every sufficiently small $\varepsilon>0$. By (2), $f$ is not Li-Yorke chaotic and thus have zero entropy. So every $\omega$-limit set of $f$ is finite by Theorem 2(1).

\section{Proof of Theorem 3}

Put $\mathcal{A}^{t}=\{0,1\}^{t}$ for $t \geq 1, \mathcal{A}^{0}=\{o\}$, and $\mathcal{A}^{*}=\bigcup_{t} \mathcal{A}^{t}$. We say that a system $\mathcal{K}=\left\{K_{a}: a \in \mathcal{A}^{*}\right\}$ of disjoint non-degenerate closed subintervals $K_{a}=\left[y_{a}, z_{a}\right]$ of $I$ is admissible if the following hold:

(1) $K_{o}=I$

(2) $y_{a}=y_{a 0}<y_{a 1}<z_{a 0}<z_{a 1}=z_{a}$ for every $a \in \mathcal{A}^{*}$;

(3) $\nu_{t}=\max \left\{\operatorname{diam} K_{a}: a \in \mathcal{A}^{t}\right\}$ converges to 0 as $t \rightarrow \infty$.

Put $Q_{t}=\bigsqcup_{a \in \mathcal{A}^{t}} K_{a}$ and $Q=Q(\mathcal{K})=\bigcap Q_{t}$; note that $Q$ is a Cantor set.

Lemma 28. Let $\mathcal{K}=\left\{K_{a}: a \in \mathcal{A}^{*}\right\}$ be admissible. Then there is a Li-Yorke non-chaotic continuous map $f: I \rightarrow I$ of type $2^{\infty}$ such that $Q(\mathcal{K})$ is the only infinite $\omega$-limit set of $f$. Moreover, $f\left(K_{a}\right)=K_{a+1}$ for every $a \in \mathcal{A}^{*}$.

Every map $f$ with properties from the lemma will be called associated to $\mathcal{K}$.

Proof. Let $\tilde{\mathcal{K}}=\left\{\tilde{K}_{a}=\left[\tilde{y}_{a}, \tilde{z}_{a}\right]: a \in \mathcal{A}^{*}\right\}$ be the (admissible) system of intervals defining the Cantor ternary set; that is, $\tilde{y}_{a 1}=\tilde{y}_{a}+(1 / 3)\left(\tilde{z}_{a}-\tilde{y}_{a}\right)$ and $\tilde{z}_{a 0}=\tilde{z}_{a}-(1 / 3)\left(\tilde{z}_{a}-\tilde{y}_{a}\right)$ for every $a \in \mathcal{A}^{*}$. Let $h$ be an increasing homeomorphism of $I$ such that $h\left(K_{a}\right)=\tilde{K}_{a}$ for every $a$. Define $f=h^{-1} \circ \tilde{f} \circ h$, where $\tilde{f}$ is Delahaye's map (see [2] or [11, Example 5.56]). Since $f$ is conjugate to $\tilde{f}$, the dynamical properties of $f$ are the same as those of $\tilde{f}$. Further, $f\left(K_{a}\right)=h^{-1}\left(\tilde{f}\left(\tilde{K}_{a}\right)\right)=h^{-1}\left(\tilde{K}_{a+1}\right)=K_{a+1}$ for every $a$.

Lemma 29. Let $f: I \rightarrow I$ be associated to an admissible system $\mathcal{K}=\left\{K_{a}=\left[y_{a}, z_{a}\right]: a \in \mathcal{A}^{*}\right\}$, and $x \in I$ be such that $\omega_{f}(x)=Q(\mathcal{K})$. Let $t \in \mathbb{N}$ and $\varepsilon>0$ satisfy

$$
\operatorname{diam}\left(K_{a}\right) \leq \varepsilon \leq \operatorname{dist}\left(K_{a}, K_{b}\right) \quad \text { for every } a \neq b \text { from } \mathcal{A}^{t}
$$

Then $\operatorname{rdet}_{m}(x, \varepsilon)=1$ for every $m \in \mathbb{N} \cup\{\infty\}$.

Proof. By (5.1),$N_{m}^{\circ}(x, t, \varepsilon)=N_{m}(x, t, \varepsilon)=\#\left\{(a, a): a \in \mathcal{A}^{t}\right\}=2^{t}$ for every $m$. Hence $\operatorname{rdet}_{m}(x, \varepsilon)=1$ by Lemma 14(5).

Lemma 30. Let $f: I \rightarrow I$ be associated to an admissible system $\mathcal{K}=\left\{K_{a}=\left[y_{a}, z_{a}\right]: a \in \mathcal{A}^{*}\right\}$, and $x \in I$ be such that $\omega_{f}(x)=Q(\mathcal{K})$. Let $t>q \geq 1$ and $\varepsilon>0$ satisfy

$$
\operatorname{diam}\left(K_{a}\right) \leq \varepsilon \leq \operatorname{dist}\left(K_{1^{t-1} 0}, K_{1^{t}}\right) \quad \text { for every } a \in \mathcal{A}^{t-q} \backslash\left\{1^{t-q}\right\} .
$$

Then $\overline{\operatorname{rdet}}_{\infty}(x, \varepsilon) \leq 2^{1-q}$. 
Proof. For any distinct $a, b \in \mathcal{A}^{t}$ we have $\operatorname{dist}_{\infty}\left(K_{a}, K_{b}\right) \geq \varepsilon$. (To see this, take $i \in\left[0,2^{t}\right)$ such that $a+i=1^{t}$; since $b+i \neq a+i$, it holds that $\operatorname{dist}_{\infty}\left(K_{a}, K_{b}\right) \geq \operatorname{dist}\left(K_{1^{t}}, K_{b+i}\right) \geq \varepsilon$ by (5.2).) Thus $N_{\infty}(x, t, \varepsilon) \leq 2^{t}$.

Take arbitrary $a \in \mathcal{A}^{t-q} \backslash\left\{1^{t-q}\right\}$ and any $b, c \in \mathcal{A}^{t} \cap[a]$. Then $\operatorname{diam}\left(K_{b} \cup K_{c}\right) \leq \operatorname{diam} K_{a} \leq \varepsilon$ and hence $N_{1}^{\circ}(x, t, \varepsilon) \geq\left(2^{t-q}-1\right) \cdot\left(2^{q}\right)^{2}$. By Lemma 14(5),

$$
\overline{\operatorname{rdet}}_{\infty}(x, \varepsilon) \leq \frac{2^{t}}{\left(2^{t-q}-1\right) \cdot\left(2^{q}\right)^{2}} \leq \frac{1}{2^{q} \cdot\left(1-2^{q-t}\right)} \leq 2^{1-q} .
$$

Proof of Theorem 3. Put $t_{1}=1$ and, for every $n \geq 1, t_{n}^{\prime}=t_{n}+(n+1)$ and $t_{n+1}=t_{n}^{\prime}+1$. We are going to inductively define an admissible system $\mathcal{K}=\left\{K_{a}: a \in \mathcal{A}^{*}\right\}$; we will implicitly assume that all intervals $K_{a}$ are defined in such a way that (2) from the definition of admissibility is satisfied. Put $K_{o}=[0,1]$, $K_{0}=[0,1 / 3]$, and $K_{1}=[2 / 3,1] ;$ then we have (5.1) with $t=t_{1}$ and $\varepsilon=\varepsilon_{1}=1 / 3$.

Assume that, for some $n \in \mathbb{N}$ and $t=t_{n}$, we have already defined $K_{a}$ for every $a$ with $|a| \leq t$. Put $\varepsilon_{n}=\max \left\{\operatorname{diam} K_{a}: a \in \mathcal{A}^{t}\right\}, \delta=\operatorname{diam} K_{1^{t}}$, and take positive $\varepsilon_{n}^{\prime}<\delta / n$. For every $b \in \mathcal{A}^{t+1}$ define $K_{b}$ in such a way that diam $K_{b}<\varepsilon_{n}^{\prime}$ if $b \neq 1^{t+1}$, and $\operatorname{diam} K_{b}>n \varepsilon_{n}^{\prime}$ if $b=1^{t+1}$. For $t+1<s \leq t+n+1=t_{n}^{\prime}$ and $b \in \mathcal{A}^{s}$ define $K_{b}$ arbitrarily requiring only that $\operatorname{dist}\left(K_{1^{s-1} 0}, K_{1^{s}}\right)>\varepsilon_{n}^{\prime}$. In this way we obtain that (5.2) is satisfied with $t=t_{n}^{\prime}, q=n$, and $\varepsilon=\varepsilon_{n}^{\prime}$.

For $t=t_{n+1}^{\prime}$ put $\varepsilon_{n+1}=(1 / 3) \min \left\{\operatorname{diam} K_{a}: a \in \mathcal{A}^{t-1}\right\}$ and define $K_{b}\left(b \in \mathcal{A}^{t}\right)$ in such a way that $\operatorname{diam} K_{b}=\varepsilon_{n+1}$. Then (5.1) is satisfied with $t=t_{n+1}^{\prime}$ and $\varepsilon=\varepsilon_{n+1}$.

In this way we obtain an admissible system $\mathcal{K}=\left\{K_{a}=\left[y_{a}, z_{a}\right]: a \in \mathcal{A}^{*}\right\}$ and sequences $\left(\varepsilon_{n}\right)_{n},\left(\varepsilon_{n}^{\prime}\right)_{n}$ decreasing to zero such that, for every $n$,

- (5.1) is satisfied with $t=t_{n}$ and $\varepsilon=\varepsilon_{n}$;

- (5.2) is satisfied with $t=t_{n}^{\prime}, q=n$, and $\varepsilon=\varepsilon_{n}^{\prime}$.

Let $f: I \rightarrow I$ be associated to $\mathcal{K}$. Put $C=Q(\mathcal{K})$ and take any $x \in C$. By Lemma 4(2) and the fact that $Q(\mathcal{K})$ is totally disconnected, $C$ is equal to the set $C$ from Lemma 4 and $\omega_{f}(x)=C$. Thus, by Lemmas 29 and 30. $\operatorname{rdet}_{\infty}\left(x, \varepsilon_{n}\right)=1$ and $\overline{\operatorname{rdet}}_{\infty}\left(x, \varepsilon_{n}^{\prime}\right) \leq 2^{1-n}$ for every $n$. This proves Theorem 3 .

\section{ACKNOWLEDGEMENTS}

Substantive feedback from Michaela Mihoková is gratefully acknowledged. This research is an outgrowth of the project "SPAMIA", MŠ SR-3709/2010-11, supported by the Ministry of Education, Science, Research and Sport of the Slovak Republic, under the heading of the state budget support for research and development. The author also acknowledges support from VEGA 1/0786/15 and APVV-15-0439 grants.

\section{REFERENCES}

[1] A. M. Blokh. The spectral decomposition for one-dimensional maps. In Dynamics reported, pages 1-59. Springer, 1995.

[2] J.-P. Delahaye. Fonctions admettant des cycles dordre nimporte quelle puissance de 2 et aucun autre cycle. CR Acad. Sci. Paris Sér. AB, 291(4), 1980.

[3] J. P. Eckmann, S. O. Kamphorst, and D. Ruelle. Recurrence plots of dynamical systems. Europhys. Lett., 4(9):973-977, 1987.

[4] P. Faure and H. Korn. A new method to estimate the kolmogorov entropy from recurrence plots: its application to neuronal signals. Phys. D, 122(1):265-279, 1998.

[5] P. Faure and A. Lesne. Recurrence plots for symbolic sequences. Internat. J. Bifur. Chaos, 20(06):1731-1749, 2010.

[6] P. Faure and A. Lesne. Estimating kolmogorov entropy from recurrence plots. In Recurrence Quantification Analysis, pages 45-63. Springer, 2015.

[7] M. Grendár, J. Majerová, and V. Špitalský. Strong laws for recurrence quantification analysis. Internat. J. Bifur. Chaos, 23(08):1350147, 2013.

[8] J. Majerová. Correlation integral and determinism for a family of $2^{\infty}$ maps. Discrete Contin. Dyn. Syst., 36(9):5067-5096, 2016.

[9] Y. B. Pesin. On rigorous mathematical definitions of correlation dimension and generalized spectrum for dimensions. J. Stat. Phys., 71(3-4):529-547, 1993.

[10] S. Ramdani, F. Bouchara, J. Lagarde, and A. Lesne. Recurrence plots of discrete-time gaussian stochastic processes. Phys. D, 330:17-31, 2016.

[11] S. Ruette. Chaos on the interval, University Lecture Series, 67. American Mathematical Society, Providence, RI, 2017.

[12] D. Schultz, S. Spiegel, N. Marwan, and S. Albayrak. Approximation of diagonal line based measures in recurrence quantification analysis. Phys. Lett. A, 379(14):997-1011, 2015.

[13] J. Smítal. Chaotic functions with zero topological entropy. Trans. Amer. Math. Soc., 297(1):269-282, 1986. 
[14] V. Špitalský. Local correlation entropy. arXiv:1612.02592, 2016.

[15] M. Thiel, M. C. Romano, and J. Kurths. Analytical description of recurrence plots of white noise and chaotic processes. Appl. Nonlinear Dynam., 11(3):20-30, 2003.

[16] C. L. Webber Jr and N. Marwan. Recurrence quantification analysis: theory and best practices. Springer, 2015.

[17] J. P. Zbilut and C. L. Webber. Embeddings and delays as derived from quantification of recurrence plots. Phys. Lett. A, 171(3-4):199-203, 1992.

[18] Y. Zou, M. Thiel, M. C. Romano, and J. Kurths. Analytical description of recurrence plots of dynamical systems with nontrivial recurrences. Internat. J. Bifur. Chaos, 17(12):4273-4283, 2007.

Slovanet A.S., Záhradnícka 151, Bratislava, Slovakia

E-mail address: vladimir.spitalsky@slovanet.net

Department of Mathematics, Faculty of Natural Sciences, Matej Bel University, Tajovského 40, Banská Bystrica, SLOVAKIA

E-mail address: vladimir.spitalsky@umb.sk 